\title{
A planar diagram approach to the correlation problem
}

\author{
D. Foerster, CPTMB, Université de Bordeaux I \\ 351, cours de la Liberation, F - 33405 Talence Cedex
}

\begin{abstract}
We transpose an idea of 't Hooft from its context of Yang and Mills' theory of strongly interacting quarks to that of strongly correlated electrons in transition metal oxides and show that a Hubbard model of $N$ interacting electron species reduces, to leading order in $N$, to a sum of almost planar diagrams. The resulting generating functional and integral equations are very similar to those of the FLEX approximation of Bickers and Scalapino. This adds the Hubbard model at large $N$ to the list of solvable models of strongly correlated electrons.
\end{abstract}

PACS Numbers: 71.27.+a 71.10.-w 71.10.Fd

\section{INTRODUCTION}

Recent numerical [1] and analytical [2] studies have shown that an extrapolation of the Hubbard model from two to $N$ distinct electron species, and with its $S U(2)$ invariance replaced by $S U(N)$, conserves the nontrivial character of this model and this provides a motivation for further study of this extrapolation. Actually, the mere fact that $S U(N)$ antiferromagnets have spin dimer or "RVB" ground states at $N=\infty$ [3], 朋] is by itself reason enough for studying the $S U(N)$ extrapolation of the Hubbard model.

Existing methods of treating such large $N$ limits are of two kinds. The first is a quasi classical saddle point method used in the context of nonlinear sigma models [5] and slave operator constraints [6] and that was applied already to the $S U(N)$ Hubbard model [7], but without conclusive results, in the opinion of this author. The second approach is 't Hooft's topological expansion [8] as applied to a $S U(N)$ deformation of the Yang-Mills theory of strong interactions. In this latter approach, $N$ fermions interact with $N^{2}$ bosons and a quasi classical or saddle point interpretation is therefore impossible.

't Hooft's topological large $N$ expansion is less well known, but more profound than the large $N$ saddle point method. In this paper we will use it to show that the $S U(N)$ Hubbard model is solvable, for $N \gg 1$, in terms of planar diagrams that can be summed via coupled integral equations.

Remarkably, our generating functional and associated integral equations turn out be very similar to those of the "fluctuation exchange approximation" (FLEX) of Bickers and Scalapino [9].

\section{A TOPOLOGICAL CLASSIFICATION OF DIAGRAMS}

We consider the Hubbard model [10] that epitomizes the problem of strongly correlated electrons (see [11] for a review) and extend it from 2 to $N$ species of electrons [7]:

$$
H=\sum_{x, y} t_{x y} \psi_{\alpha}^{*}(x) \psi_{\alpha}(y)+\frac{U}{N} \sum_{x}\left(\sum_{\alpha=1 . . N} \psi_{\alpha}^{*}(x) \psi_{\alpha}(x)\right)^{2}
$$

We have replaced $U \rightarrow \frac{U}{N}$ to have a stable limit as $N$ varies and to regain the conventional meaning of $U$ at $N=2$. A suitable chemical potential is implicit in all our expressions.

A satisfactory treatment of the interaction between electrons and their own spin and density fluctuations is at the heart of the correlation problem. In the model of Hubbard (and in essentially any electronic system with finite range two body potentials) this interaction can be made explicit by means of a Hubbard-Stratonovich transformation:

$$
\begin{aligned}
& \int D \phi_{\alpha \beta} \exp -\int_{0}^{\beta} \sum_{\alpha, \beta, x}\left(\phi_{\alpha \beta}^{*} \phi_{\alpha \beta}+\sqrt{\frac{U}{N}} \psi_{\alpha}^{*} \phi_{\alpha \beta} \psi_{\beta}+\text { h.c. }\right) d \tau \\
= & \operatorname{const}(T) \exp -\frac{U}{N} \int_{0}^{\beta}\left(\sum_{\alpha, \beta, x} \psi_{\alpha}^{*} \psi_{\alpha} \psi_{\beta}^{*} \psi_{\beta}+\text { density }\right) d \tau
\end{aligned}
$$

Here "density" stands for terms $\sim \psi_{\alpha}^{*} \psi_{\alpha}$ that can be absorbed in a redefinition of the chemical potential and which we may therefore ignore. To avoid a proliferation of unphysical fields, we impose $\phi_{\alpha \beta}^{*}=\phi_{\beta \alpha}$. We then find the following bare correlators for $\phi_{\alpha \beta}$ and $\psi_{\alpha}$ 


$$
\begin{aligned}
& <\phi_{\alpha \beta}(1) \phi_{\alpha^{\prime} \beta^{\prime}}^{*}(2)>_{0}=\frac{1}{2} \delta_{\alpha \alpha^{\prime}} \delta_{\beta \beta^{\prime}} \delta(1,2) \text { and }<\phi_{\alpha \beta} \phi_{\alpha^{\prime} \beta^{\prime}}>_{0}=\frac{1}{2} \delta_{\alpha \beta^{\prime}} \delta_{\beta \alpha^{\prime}} \delta(1,2) \\
& <\psi_{\alpha}(1) \psi_{\beta}^{*}(2)>_{0}=\delta_{\alpha \beta}\left(\frac{1}{\partial_{\tau}+t_{x y}}\right)(1,2)
\end{aligned}
$$

where $\delta(1,2)$ is a shorthand for $\delta\left(\tau_{1}-\tau_{1}\right) \delta \vec{x}_{1}, \vec{x}_{2}$. With the help of the spin and density fluctuations $\phi_{\alpha \beta}$ the partition function of the repulsive Hubbard model can be written as

$$
\begin{aligned}
& Z=\int D \phi D \psi \exp -\int_{0}^{\beta} d t\left(\sum_{\alpha, \beta, x}\left(\psi_{\alpha}^{*} \partial_{t} \psi_{\alpha}+\phi_{\alpha \beta}^{*} \phi_{\alpha \beta}\right)+H\right) \\
& H=2 \sqrt{\frac{U}{N}} \sum_{x, \alpha, \beta} \psi_{x \alpha}^{*} \phi_{x, \alpha \beta} \psi_{x \beta}+\sum_{x, y, \alpha} t_{x y} \psi_{x \alpha}^{*} \psi_{y \alpha}
\end{aligned}
$$

From eqs (3, 4) the perturbative diagrams associated with the above Hamiltonian may be drawn in terms of single line propagators for electrons, double line propagators for the bosons and a three point vertex $2 \sqrt{\frac{U}{N}} \psi_{\alpha}^{*} \phi_{\alpha \beta} \psi_{\beta}$ at which a particle hole excitation splits into its constituents, see figure(1).

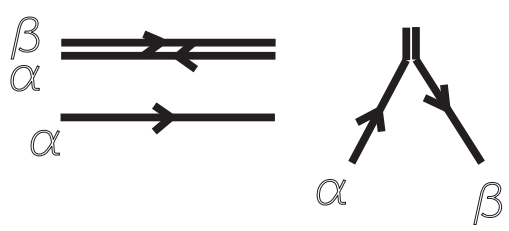

FIG. 1. Bare propagators and bare vertex in the $S U(N)$ Hubbard model

Using an argument that 't Hooft originally applied to an $S U(N)$ deformation of Quantum Chromodynamics [8], we now show that any perturbative diagram made up of such propagators and vertices carries a weight $N^{\chi}$ where $\chi=S_{2}-S_{1}+S_{0}$ is the Euler characteristic [12] of its associated topological surface. Here $S_{0}$ stands for the number of vertices, $S_{1}$ for the number of propagators and $S_{2}$ for the number of "area like" pieces associated with each diagram. To define an "area like" piece, we follow an index line until it closes. The topological surface associated with each diagram then consists of a collection of loops that are glued together along segments that belong to double lines representing boson propagators. In fact, this is precisely the type of representation of a surface that is used in elementary topology [12] where for instance a torus is represented in terms of a rectangle with identified opposite sides.

By definition then, each closed index loop of a given diagram contributes one unit to $S_{2}$. We may count the endpoints of each propagator either from the propagator point of view $\left(2 S_{1}\right)$ or from the vertex point of view $\left(3 S_{0}\right)$ to obtain the topological relation $S_{1}=\frac{3}{2} S_{0}$. With a factor $\sqrt{\frac{U}{N}}$ from each vertex and with a factor $N$ from each closed index loop, a diagram carries a weight factor of

$$
\text { weight }=U^{\frac{S_{0}}{2}} N^{S_{2}-\frac{S_{0}}{2}} \stackrel{S_{1}=\frac{3}{2} S_{0}}{=} U^{\frac{S_{0}}{2}} N^{\chi}
$$

The Euler characteristic of an orientable closed surfaces with $h$ handles and $o$ openings is $\chi=2-2 h-o$. The last equation implies that the "planar" diagrams that can be drawn in a plane without intersections of lines are the important ones, while extra openings and handles are suppressed by factors of $\frac{1}{N}$ and $\frac{1}{N^{2}}$, respectively.

Although it is difficult to intuitively understand the intrinsic topology of the topological surface associated with any given diagram, it is easy to draw a surface with a minimal number of handles and holes on which a given diagram can be drawn. Its intrinsic geometry and Euler characteristic coincide with that of the topological surface defined via single and double line propagators and vertices. We illustrate this with a nonleading contribution to the electron self energy in figure (2) where the red lines represent boson propagators and the black line an electron. 


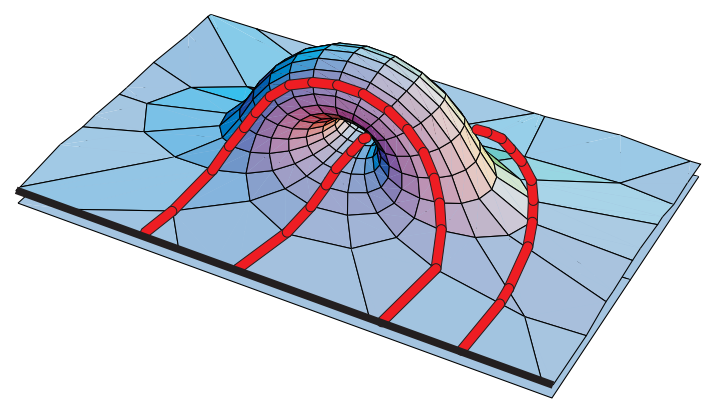

FIG. 2. Nonplanar and therefore nonleading contribution to the fermion self energy

The extra handle is needed here for the boson propagators not to cross each other. From the factor $N^{\chi}$ of eq(5) and the expression for the Euler characteristic we see that this diagram is suppressed, relative to the planar ones (and without crossings of boson propagators) by a factor of $\frac{1}{N^{2}}$. Of course we arrive at the same conclusion also by carefully drawing this diagram in terms of lines and double lines and by counting powers of $N$ from vertices and index loops.

Finally, we must also mention a difficulty in the present $S U(N)$ extrapolation of the Hubbard model. The diagram of figure (3) without the large half circle contributes to the bosonic self energy and gives rise to scalar or trace contribution $\sim \frac{\delta_{\alpha \beta} \delta_{\alpha^{\prime} \beta^{\prime}}}{N^{2}}$ in the $<\phi_{\alpha \beta} \phi_{\alpha^{\prime} \beta^{\prime}}^{*}>$ propagator.

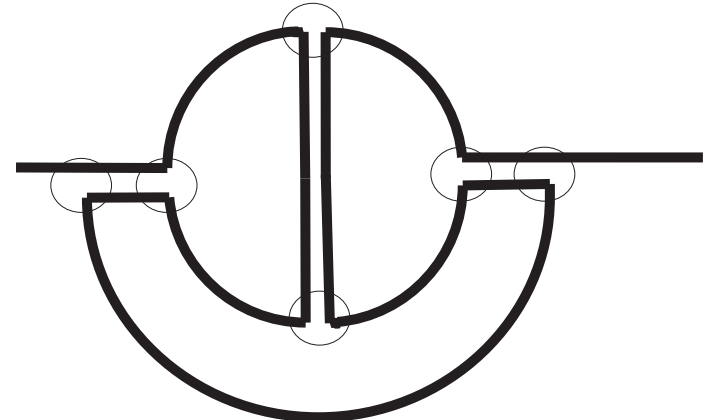

FIG. 3. Self energy correction due to a scalar part in the boson propagator

This means that the tensor character of the full boson propagator is different from that of the bare one in eq(3). Luckily, this term is suppressed at large $N$, as we shall see. The small circles in figure (3) indicate vertices, of which there are six (for the electron self energy) with a factor of $\sqrt{\frac{U}{N}}$ each and hence this diagram gives a contribution of order $O\left(\frac{1}{N^{3}}\right)$ to the electron self energy. We shall, therefore, ignore the scalar or trace part of the $<\phi \phi^{*}>$ propagator on the grounds that (i) its effects are small at large $N$ and (ii) because it represents only uninteresting charge density fluctuations [13].

\section{COUPLED INTEGRAL EQUATIONS AND GENERATING FUNCTIONAL}

Expanding the cubic interaction in eq(14) to second order (or by appealing to standard diagram lore explained in textbooks [14]) we find the following lowest order electronic self energy

$$
\begin{aligned}
& \Sigma_{\alpha_{1} \alpha_{2}}(1,2)=\frac{4 U}{N}<\varphi_{\alpha_{1} \alpha_{3}}(1) \psi_{\alpha_{3}}(1) \cdot \psi_{\alpha_{4}}^{*}(2) \phi_{\alpha_{4} \alpha_{2}}(2)>=\frac{4 U}{N} D_{\alpha_{1} \alpha_{3} \mid \alpha_{4} \alpha_{2}}(1,2) G_{\alpha_{3} \alpha_{4}}(1,2) \\
& G_{\alpha_{3} \alpha_{4}}(1,2) \equiv<T\left\{\psi_{\alpha_{3}}(1) \psi_{\alpha_{4}}^{*}(2)\right\}>\text { and } D_{\alpha_{1} \alpha_{3} \mid \alpha_{4} \alpha_{2}}(1,2) \equiv<T\left\{\varphi_{\alpha_{1} \alpha_{3}}(1) \phi_{\alpha_{4} \alpha_{2}}(2)\right\}>
\end{aligned}
$$

We promote this expression to the complete skeleton one by using full propagators in it. This, however, ignores vertex corrections. The first vertex correction to the electronic self energy $\Sigma$ is suppressed by a factor of $\frac{1}{N}$ and may be ignored at leading order in $N$.

A more graphic way of arriving at eq(6) is by considering the sum of planar or "rainbow like" contributions to the electronic self energy and by removing the outermost scalar propagator or rainbow. 
To exploit eq(6) we use the tensor structure of $D$ and $G$ which is (ignoring the scalar or trace contribution to $D$ )

$$
\begin{aligned}
D(1,2)_{\alpha_{1} \alpha_{3} \mid \alpha_{4} \alpha_{2}} & =\delta_{\alpha_{1} \alpha_{2}} \delta_{\alpha_{3} \alpha_{4}} D(1,2) \text { and } \Pi(1,2)_{\alpha_{1} \alpha_{3} \mid \alpha_{4} \alpha_{2}}=\delta_{\alpha_{1} \alpha_{2}} \delta_{\alpha_{3} \alpha_{4}} \Pi(1,2) \\
G_{\alpha_{3} \alpha_{4}}(1,2) & =\delta_{\alpha_{3} \alpha_{4}} G(1,2) \text { and } \Sigma_{\alpha_{1} \alpha_{2}}(1,2)=\Sigma(1,2) \delta_{\alpha_{1} \alpha_{2}}
\end{aligned}
$$

and we find

$$
\Sigma(1,2)=4 U D(1,2) G(1,2)
$$

Starting again from second order perturbation theory for $\Pi$ and promoting it to a skeleton diagram (which is correct to leading order in $N$ ) we find a boson self energy of

$$
\begin{aligned}
\Pi_{\alpha_{1} \beta_{1} \mid \alpha_{2} \beta_{2}}(1,2) & =\frac{4 U}{N}<\psi_{\alpha_{1}}^{*}(1) \psi_{\beta_{1}}(1) \psi_{\alpha_{2}}^{*}(2) \psi_{\beta_{2}}(2)>=-\frac{4 U}{N} \delta_{\alpha_{1} \beta_{2}} \delta_{\alpha_{2} \beta_{1}} G(1,2) G(2,1) \\
\Pi(1,2) & =-\frac{4 U}{N} G(1,2) G(2,1)
\end{aligned}
$$

where the minus sign reflects the fermion loop in this diagram. To be sure of the self consistency of the integral equations for $\Sigma$ and $\Pi$ and, at a later stage, for thermodynamics and linear response, we need a functional that generates the equations for $\Sigma$ and $\Pi$. To find it, we consider the lowest order diagram for the logarithm of the partition function and promote it also to a skeleton diagram:

$$
\begin{aligned}
F & =\frac{2 U}{N} \sum<\psi_{\alpha_{1}}^{*}(1) \Phi_{\alpha_{1} \alpha_{2}}(1) \psi_{\alpha_{2}}(1) \psi_{\alpha_{3}}^{*}(2) \Phi_{\alpha_{3} \alpha_{4}}(2) \psi_{\alpha_{4}}(2)> \\
& =-\frac{2 U}{N} \sum D_{\alpha_{1} \alpha_{2} \mid \alpha_{3} \alpha_{4}}(1,2) G_{\alpha_{2} \alpha_{3}}(1,2) G_{\alpha_{4} \alpha_{1}}(2,1)
\end{aligned}
$$

Here $\sum$ stands for a sum over repeated indices and for integration over Matsubara time and the extra factor $1 / 2$ is due to the symmetry of this diagram. Combining eqs(7,10) one recognizes that $F$ contains two closed index loops and one obtains

$$
F=-2 U \sum D(1,2) G(1,2) G(2,1)
$$

with full Green's functions $D, G$. By general field theory or many body lore [15] our previously obtained equations for $\Sigma, \Pi$ should come out by varying $F$ with respect to $D, G$ :

$$
\begin{aligned}
& \Sigma(1,2)=-\frac{\delta}{\delta G(2,1)} \sum_{1^{\prime} 2^{\prime}}-2 U D\left(1^{\prime}, 2^{\prime}\right) G\left(1^{\prime}, 2^{\prime}\right) G\left(2^{\prime}, 1^{\prime}\right) \\
& \Pi(1,2)=\frac{2}{N} \frac{\delta}{\delta D(2,1)} \sum_{1^{\prime} 2^{\prime}}-2 U D\left(1^{\prime}, 2^{\prime}\right) G\left(1^{\prime}, 2^{\prime}\right) G\left(2^{\prime}, 1^{\prime}\right)
\end{aligned}
$$

(diagrammatically speaking, functional differentiation applied to vacuum diagrams opens propagator lines in all possible ways). Dyson's equations $G^{-1}=G_{0}^{-1}-\Sigma$ and $D^{-1}=D_{0}^{-1}-\Pi$ for the self energy may also be written in differential form:

$$
\begin{aligned}
& \Sigma(1,2)=-\frac{\delta}{\delta G(2,1)} \operatorname{Tr}\left(\log G-G / G_{0}\right) \\
& \Pi(1,2)=-\frac{\delta}{\delta D(2,1)} \operatorname{Tr}\left(\log D-D / D_{0}\right)
\end{aligned}
$$

Taken together, eqs $(12,13)$ permit the introduction of a new functional $\Omega$ that is stationary with respect to variations in $G$ and $D$ :

$$
\begin{aligned}
\Omega & =\operatorname{Tr}\left(\log G-G / G_{0}\right)-\frac{N}{2} \operatorname{Tr}\left(\log D-D / D_{0}\right)+\sum_{1,2} 2 U D(1,2) G(1,2) G(2,1) \\
\frac{\delta \Omega}{\delta G} & =\frac{\delta \Omega}{\delta D}=0
\end{aligned}
$$

That $\Omega$ represents the logarithm of the partition function of the system under consideration can be proved by generalizing the arguments of Luttinger and Ward 15] to coupled bosons and fermions. The factor $N / 2$ reflects the presence, in our system, of $N^{2}$ real bosons as compared to only $N$ complex fermions. 


\section{THE PHYSICAL MEANING OF THE INTEGRAL EQUATIONS}

To gain a first understanding of the integral equations (8,9) one may solve them iteratively in powers of $1 / N$. From eqs (8, 9) we see that one may set $G=G_{0}$ to leading order and then one finds

$$
\begin{aligned}
G & =G_{0}+O\left(\frac{1}{N}\right) \\
\Pi(1,2) & =-\frac{4 U}{N} G_{0}(1,2) G_{0}(2,1)+O\left(\frac{1}{N^{2}}\right)
\end{aligned}
$$

It is now clear that $\Pi$ represents paramagnon type spin fluctuations (see [16] for an appraisal of such theories and for further references) and we must compare with the FLEX approximation of Bickers and Scalapino [9] that was designed to deal with particle hole fluctuations in correlated systems and, in particular, with spin fluctuations in the Hubbard model.

Since the FLEX approximation is stated most succinctly in terms of a free energy or generating functional we compare with [17] where the FLEX approximation to the free energy of the Hubbard model is written down explicitly. The first thing we notice from [17] is that the FLEX functional involves only $G$ but not $D$. To compare our functional with the FLEX one, we must eliminate $D$ from it by using the saddle point condition eq(9). Together with Dyson's equation for $D$, this leads to a drastic simplification of the functional $\Omega$ :

$$
[\Omega]_{\frac{\delta \Omega}{\delta D}=0}=\mathrm{const}+\operatorname{Tr}\left(\log G-G / G_{0}\right)-\frac{N}{2} \operatorname{Tr}(\log D)
$$

where $D^{-1}=D_{0}^{-1}-\Pi$. Readers who are suspicious of the simplicity of this functional may be reassured by the fact that a functional that includes the first subleading order (and which is not given here) has a more subtle mathematical structure.

To compare the functional of the last equation with that of FLEX we note that according to [17] the FLEX functional contains no linear term in $\Pi$, while ours does (when expanding in $\Pi$ or $1 / N$ ) so the two functionals do not coincide. Another difference is that FLEX violates crossing symmetry [18] while the large $N$ approach does not.

Such differences between the two approaches are not surprising in view of the fact that the Kadanoff-Baym prescription for writing down conserving approximations is non unique 15] while there is no ambiguity in the large $N$ approach as described here, except that one may mix spin and charge fluctuations with an arbitrary mixing angle. This mixing angle ceases to be arbitrary only if any one of the fluctuating fields develops an expectation value. However, because we do not expect any condensation of local pairs $\psi_{\alpha}(x) \psi_{\beta}(x)$ in the repulsive Hubbard model, there is no reason to introduce such a mixing of channels in the present context.

\section{CONCLUSIONS}

Our main result is the observation that the $S U(N)$ extension of the Hubbard model can be solved, at large $N$, by a transcription of 't Hooft's planar diagram technique from $S U(N)$ Yang-Mills theory to this model. At leading order in $N$ the functional $\Omega$ of eq(14) must be minimized via the saddle point equations $(8,9)$ for $\Sigma$ and $\Pi$. Because our generating functional and integral equations are quite similar to the FLEX equations of Bickers and Scalapino we expect that their solutions should be qualitatively the same.

Since the ground state of the undoped $S U(N)$ theory for large $N$ is a collection of RVB type dimers our arguments constitute a bridge between Anderson's early RVB ideas 国 and the FLEX method.

One of the shortcomings of the $S U(N)$ extrapolation of the Hubbard model presented here is that it is unlikely to describe antiferromagnetic order, such order being unnatural for more than two electron species. It is probably no coincidence that the absence of antiferromagnetic order and of a spin gap is also the main shortcoming of the FLEX approximation.

Clearly, the approach presented here must be improved in a way that permits a smoother extrapolation back to the electrons of our physical world with their two spin orientations and their (anti) ferromagnetic correlations.

\section{Acknowledgements}

I am indebted to P. Schuck and J.-M. Robin for useful correspondence and to A. Hewson, J. Keller, T. Pruschke and M. Zoelf for informative discussions. I thank T. Dahm for extensive correspondence on the FLEX approach and I am indebted to S. Villain-Guillot for numerous discussions of the manuscript. Finally I wish to acknowledge the marvelous inspiration provided by the 1999 Hvar School on Correlated Electrons. 
[1] O. Gunnarsson, Z. Physik B104 (1997) 279.

[2] R. Assaraf, P. Azaria, M. Caffarel, P. Lecheminant, Phys. Rev. B 60 (1999) 2299.

[3] N. Read and S. Sachdev, Nucl. Phys. B315 (1989) 609; D. S. Rokshar, Phys. Rev. B42 (1990) 2526 and (for $O(\infty)$ instead of $S U(\infty))$ D. Foerster and F. Triozon, Phys. Rev. B56 (1997) 8069.

[4] For details and references on the RVB idea see P.W. Anderson, "The Theory of Superconductivity in the high $T_{c}$ Cuprates" Princeton University Press, Princeton, N.J. 1997.

[5] H. E. Stanley, Phys. Rev. 176 (1968) 718. For examples of both versions of the large $N$ approach, see the reprint volume "The large $N$ Expansion in Quantum Statistical Physics", edited by E. Brezin and S. Wadia, World Scientific, Singapore 1993.

[6] for reviews see: A. C. Hewson, "The Kondo Problem to Heavy Fermions", Cambridge University Press (1993) and N. E. Bickers, Rev. Mod. Phys. 57 (1987) 845.

[7] I. Affleck, B.J. Marston, Phys. Rev. B. 39 (1989); I am indebted to P. Lecheminant for calling this paper to my attention.

[8] G. 't Hooft, Nucl. Phys. B72 (1974) 461 and Nucl. Phys. B75 (1974) 461.

[9] N. E. Bickers and D. J. Scalapino, Ann. Phys. (N. Y.) 193 (1989) 206.

[10] J. Hubbard, Proc. Roy. Soc London A 276 (1963) and A 281 (1964) 401.

[11] P. Fulde, "Electron Correlations in Molecules and Solids", Springer Series in Solid-State Sciences 100, N.Y. 1991.

[12] See for example J. Stillwell, "Classical Topology and Combinatorial Group Theory", Graduate Texts in Mathematics 72, Springer, N.Y. 1993.

[13] There is a related complication with the vertex that changes its tensor character at higher order in $1 / N$ but the corresponding contribution to the electronic self energy is suppressed by a factor of $1 / N^{2}$

[14] A. A. Abrikosdov, L. P. Gorkov and I. E. Dzyaloshinski, "Methods of Quantum Field Theory in Statistical Mechanics, Dover, N. Y. 1997.

[15] G. Baym, Phys. Rev. B 127 (1962) 1391; J. M. Luttinger and J. C. Ward, Phys. Rev. 118 (1960) 1417.

[16] Y. M. Vilk and A.M. Tremblay, J. Phys. (France) I 7 (1997) 1309.

[17] J. W. Serene and D. W. Hess, Phys. Rev. B 44 (1991) 3391.

[18] N. E. Bickers and S. R. White, Phys. Rev. B 43 (1991) 8044. 